\title{
Nitrogen-enriched carbon with extremely high mesoporosity and tunable mesopore size for high-performance supercapacitors
}

\author{
Xiaoqing Yang ${ }^{\text {a, }}$, Chengfei $\mathrm{Li}^{\text {a }}$, Ruowen $\mathrm{Fu}^{\text {b, * }}$ \\ ${ }^{a}$ School of Materials and Energy, Guangdong University of Technology, Guangzhou \\ 510006, PR China \\ ${ }^{b}$ Materials Science Institute, School of Chemistry and Chemical Engineering, Sun \\ Yat-sen University, Sun Yat-sen University, Guangzhou 510275, PR China
}

\begin{abstract}
:
As one of the most potential electrode materials for supercapacitors, nitrogen-enriched nanocarbons are still facing challenge of constructing developed mesoporosity for rapid mass transportation and tailoring their pore size for performance optimization and expanding their application scopes. Herein we develop a series of nitrogen-enriched mesoporous carbon (NMC) with extremely high mesoporosity and tunable mesopore size by a two-step method using silica gel as template. In our approach, mesopore size can be easily tailored from 4.7 to $35 \mathrm{~nm}$ by increasing the HF/TEOS volume ratio from $1 / 100$ to $1 / 4$. The NMC with mesopores of $6.2 \mathrm{~nm}$ presents the largest mesopore volume, surface area and mesopore ratio of $2.56 \mathrm{~cm}^{3} \mathrm{~g}^{-1}, 1003 \mathrm{~m}^{2} \mathrm{~g}^{-1}$ and $97.7 \%$, respectively. As a result, the highest specific capacitance of $325 \mathrm{~F} \mathrm{~g}^{-1}$ can be obtained at the current density of $0.1 \mathrm{~A} \mathrm{~g}^{-1}$, which can

* Corresponding authors

Tel: +86-020-39322570 (X. Yang); +86-020-84115112 (R. Fu);

E-mail addresses: yxq-886@163.com (X. Yang); cecfrw@mail.sysu.edu.cn (R. Fu).
\end{abstract}


stay over $88 \%\left(286 \mathrm{~F} \mathrm{~g} \mathrm{~g}^{-1}\right)$ as the current density increases by 100 times $\left(10 \mathrm{~A} \mathrm{~g}^{-1}\right)$. This approach may open the doors for preparation of nitrogen-enriched nanocarbons with desired nanostructure for numerous applications.

Keywords: Supercapacitors; Electrode materials; Nitrogen-enriched Carbon; Nanostructure; Mesopore size 


\section{Introduction}

Nowadays, nitrogen-enriched nanocarbon (NC) has been widely investigated for electrode materials of energy storage devices such as lithium-ion batteries [1-3], fuel cells $[4,5]$ and especially supercapacitors [6-16] by virtue of their stable physicalchemistry property, porous structure, large surface area and tunable nitrogen functional groups (NFGs), which can provide pseudocapacitance [11-13] and enhance the wettability of the electromaterials [13-16]. The key to their success in applications strongly depends on the ability to design well-controllable nanostructure.

Generally, postprocess $[11,12,16-18]$, for example, ammonia heat treatment and melamine immersion, is the most frequently used technique to introduce nitrogen, giving rise to a tedious process, pore structure collapse and unstable NFGs upon long-term/hardness working condition [12, 16, 19-21]. Moreover, the subsequently introduced NFGs may block pores and thereby reduce the ion-accessible surface area [18]. Although the strategies of carbonizing nitrogen-enriched precursors directly, e.g., polyacrylonitrile (PAN) and melamine-formaldehyde resin, are able to overcome these above shortcomings [19-29], they exhibit inevitable defects, such as costly procedure owing to the fabrication of expensive silica template of SBA-15 with special nanostructure [20-22], as well as uncontrollable pore size [26-28], which limits its performance optimization and application expanding. Furthermore, in many cases, the high-rate performance of supercapacitors is limited seriously due to poor mass transfer capability resulted from the micropore-dominant nanostructure [23-29]. Thus, exploring high mesoporosity with well-defined nanostructures has been a 
long-pursued goal for the development of state-of-the-art NCs, which may provide new opportunities in supercapacitor application.

We have reported previously that the preparation of nitrogen-enriched mesopore carbon (NMC) by constructing an inexpensive silica gel as a template [19]. The porosity and NFGs content of the as-prepared NMCs can be controlled in a certain extent by changing the carbonization temperature. This strategy offers a mesopore-dominant $\mathrm{NC}$ with 3D continues mesopore structure and carbon skeleton for guaranteeing rapid transportation of electrolyte/electron, especially at high current densities in supercapacitor application. Nevertheless, its large mesopore size of $23 \mathrm{~nm}$ results in relatively low surface area for electric double layer formation and low NFGs utilization. Therefore, a very important yet really challenging issue to address is how to tailor the mesopore size for nanostructure control to further improve the electrochemical performance and expand their application scopes. That is to say, one can choose an appropriate NC as one wishes for different application purposes to realize a target oriented use.

It has been proved that the shape and size of silica framework can be well controlled by hydrofluoric acid (HF) [30 31], which offers us with the possibility of obtaining controllable silica framework for controlling the mesopore size of the as-prepared NMC. As we know, an appropriately small mesopore size will increase the ion-accessible surface area for charge accumulation while guaranteeing rapid mass transport at high current loads. Based on this, a class of silica gel with controllable skeleton is prepared by varying the HF dosage and then used as the template. Its 
well-controlled and 3D continuous structure allows one to tailor the mesopore size of the resulting carbons over a wide range $(4.7-35 \mathrm{~nm})$ as well as to construct continuous mesopore structure with extremely high mesoporosity (Figure 1). As a result, the sample with a mesopore size, mesopore ratio $\left(\mathrm{V}_{\text {mes }} \%\right)$ and surface area of $6.2 \mathrm{~nm}$, $97.7 \%$ and $1003 \mathrm{~m}^{2} \mathrm{~g}^{-1}$, respectively, demonstrates the highest specific capacitance of $325 \mathrm{~F} \mathrm{~g}^{-1}$ at $0.1 \mathrm{~A} \mathrm{~g}^{-1}$, and a high capacity retention of $88 \%\left(286 \mathrm{~F} \mathrm{~g}^{-1}\right)$ can be achieved as the current density increases to $10 \mathrm{~A} \mathrm{~g}^{-1}$.

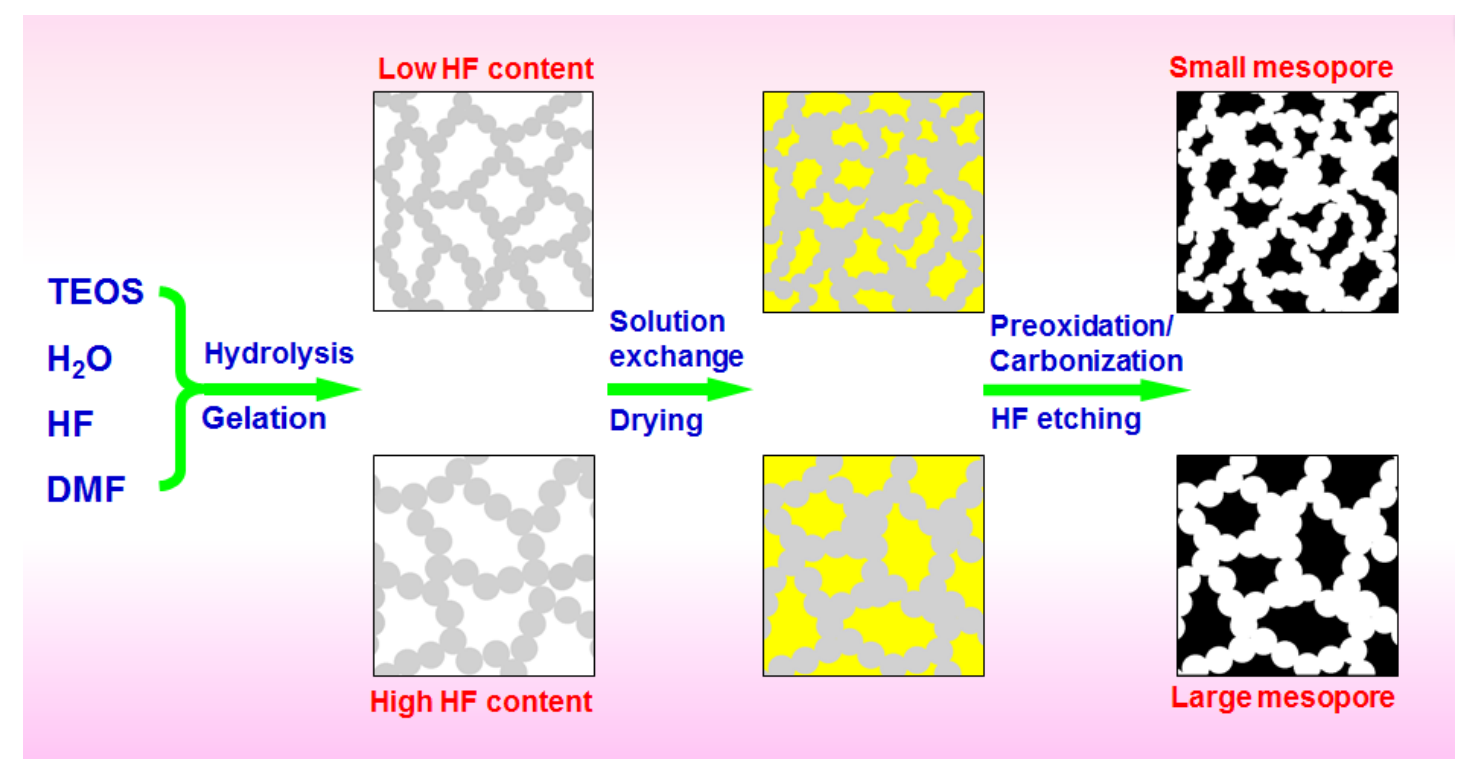

Figure 1. Schematic diagram for the preparation of NMCs with tunable mesopore size

\section{Experimental}

\subsection{Preparation of NMCs}

The fabrication of NMCs was based on our previous report via a sol-gel method with some modifications of varying the HF/ tetraethyl orthosilicate (TEOS) ratio [19]. Deionized water, TEOS and N, N-dimethylformamide (DMF) were first mixed in a polytetrafluoroethylene (PTFE) vessel at a volume ratio of 1:2:5 successively. Then a 
predetermined amount of HF solution with a concentration of $40 \mathrm{wt} \%$ was added under magnetic stirring. After complete homogenization, the obtained mixture was sealed up and gelated/aged at $90{ }^{\circ} \mathrm{C}$ for 3 days. The resulting colorless silica gel was transferred into a conical flask followed by adding PAN/DMF solution with a concentration of $0.5 \mathrm{~g}$ PAN/10 $\mathrm{mL}$ DMF $(1 \mathrm{~g}$ gel/4 $\mathrm{mL}$ solution). Then the conical flask was sealed and oscillated with a rotational speed of $180 \mathrm{r} \mathrm{min}{ }^{-1}$ at $30{ }^{\circ} \mathrm{C}$ for 2 days. After pumping filtration, drying at $90{ }^{\circ} \mathrm{C}$, the samples were preoxidation in a muffle furnace and then carbonized at $850{ }^{\circ} \mathrm{C}$ under $\mathrm{N}_{2}$ atmosphere, respectively. The as-obtained silica/nanocarbon composites were washed using excessive amount of 40 wt.\% HF solution to remove the silica, followed by filtration, washing with deionized water and drying. The as-prepared PAN-based NMCs were denoted as NMCx, where $x$ represented the TEOS/HF ratio.

\subsection{Characterization of the as-prepared NMCs}

Fourier-transform infrared reflection (FTIR) spectrum was recorded on a Shimadz IR Prestige-21 FTIR spectrometer using KBr pellet. The microstructure of the carbon samples was investigated by a transmission electron microscope (TEM, JEOL JEM-2010), scanning electron microscopy (SEM, JSM-6330F) and an ASAP 2020 surface area and porosity analyzer (Micromeritics Instrument Corporation). The Brunauer-Emmett-Teller method was utilized to calculate the BET surface area $\left(\mathrm{S}_{\mathrm{BET}}\right)$ using adsorption data in a relative pressure range from 0.05 to 0.21 . Micropore volume $\left(\mathrm{V}_{\mathrm{mic}}\right)$, mesopore volume $\left(\mathrm{V}_{\mathrm{mes}}\right)$ and mesopore size distribution of the samples were analyzed by t-plot and BJH (Barrett-Joyner-Halendar) theory, respectively. 
Nitrogen content was measured using a Vario EL CHNS Elemental Analyzer (Elementar Corporation, Germany).

\subsection{Electrochemical measurements}

The electrochemical performance of the NMC samples was measured in $1 \mathrm{M}$ $\mathrm{H}_{2} \mathrm{SO}_{4}$ using a sandwich-type two-electrode testing cell. Composite electrode comprising NMC (80 wt.\%), acetylene black (10 wt.\%) and poly (vinylidene difluoride) (PVDF, 5 wt.\%) binder was used as working and counter electrode, respectively. The mass loading of NMCs on each electrode was about $4 \mathrm{mg}$. All electrochemical measurements were performed with assembled two-electrode supercapacitors at ambient temperature. The galvanostatic charge-discharge tests were executed using a Xinwei battery test equipment (CT2001A) at different current loads between 0.1 and $10 \mathrm{~A} \mathrm{~g}^{-1}$ in a potential window of $0-1 \mathrm{~V}$. The specific gravimetric capacitance $\mathrm{C}_{\mathrm{g}}$ (in $\mathrm{F} \mathrm{g}^{-1}$ ) of $\mathrm{NMC}$ can be calculated by the formula: $C_{g}=\frac{I \times \Delta t}{\Delta U} \times$ $\frac{m_{1}+m_{2}}{m_{1} \times m_{2}}$, where $I$ is the discharge current; $\Delta t$ is the discharge time; $\Delta U$ is the discharge voltage; $m_{1}$ and $m_{2}$ are the mass of the positive and negative active electrode materials. Specific capacitance per surface area $C_{S}\left(\right.$ in $\mu \mathrm{F} \mathrm{cm}^{-2}$ ) was obtained by dividing the $\mathrm{C}_{\mathrm{g}}$

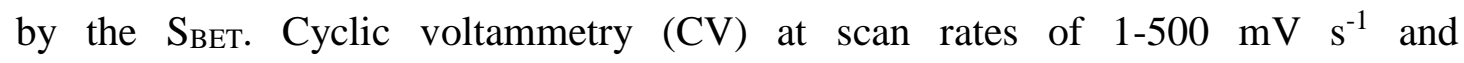
electrochemical impedance spectroscopy (excitation signal: $5 \mathrm{mV}$ and frequency range: $0.1-100,000 \mathrm{~Hz}$ ) were also recorded using an IM6ex electrochemical workstation. 


\section{Results and discussions.}
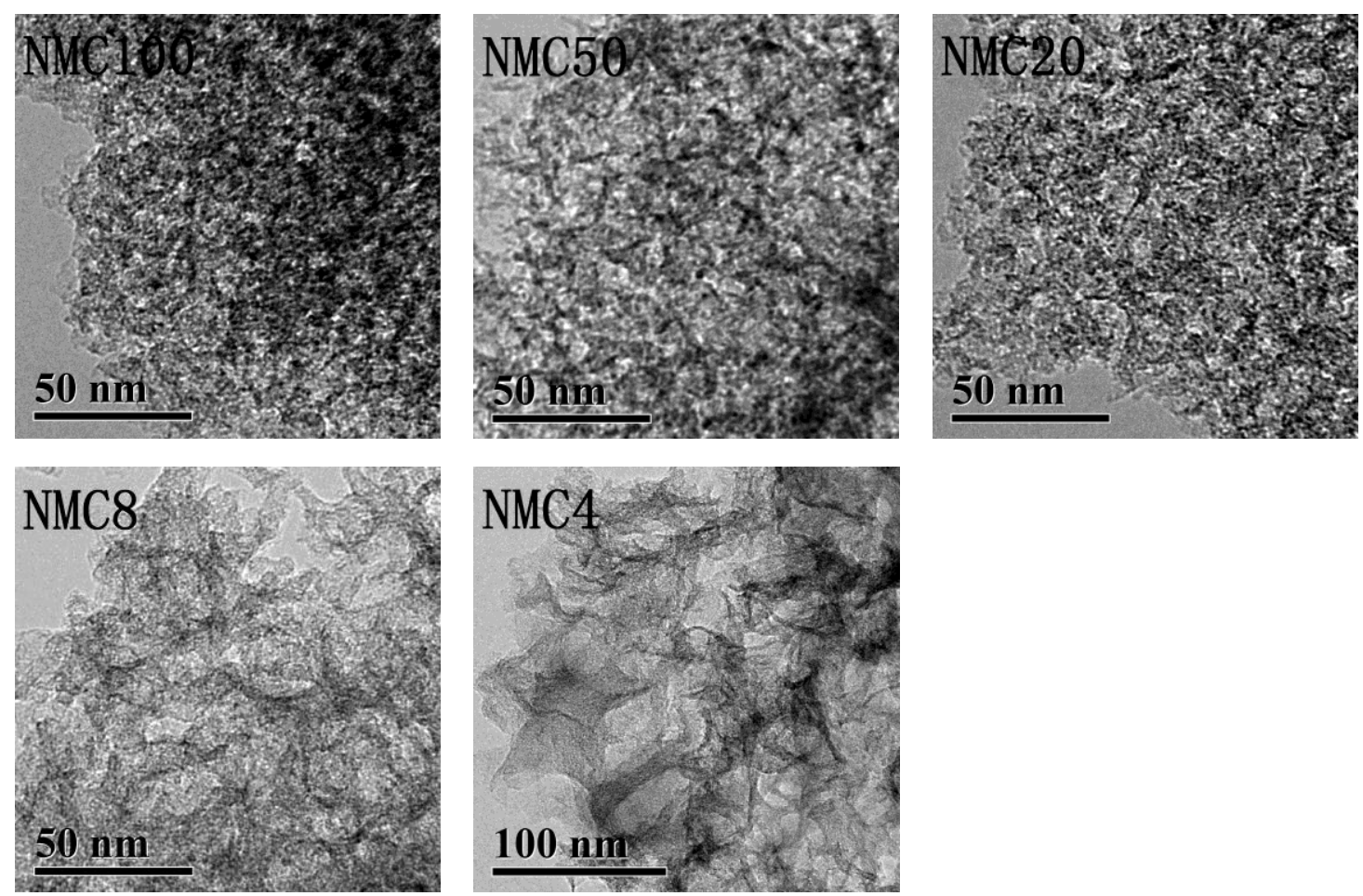

Figure 2. TEM images of the as-prepared NMCs
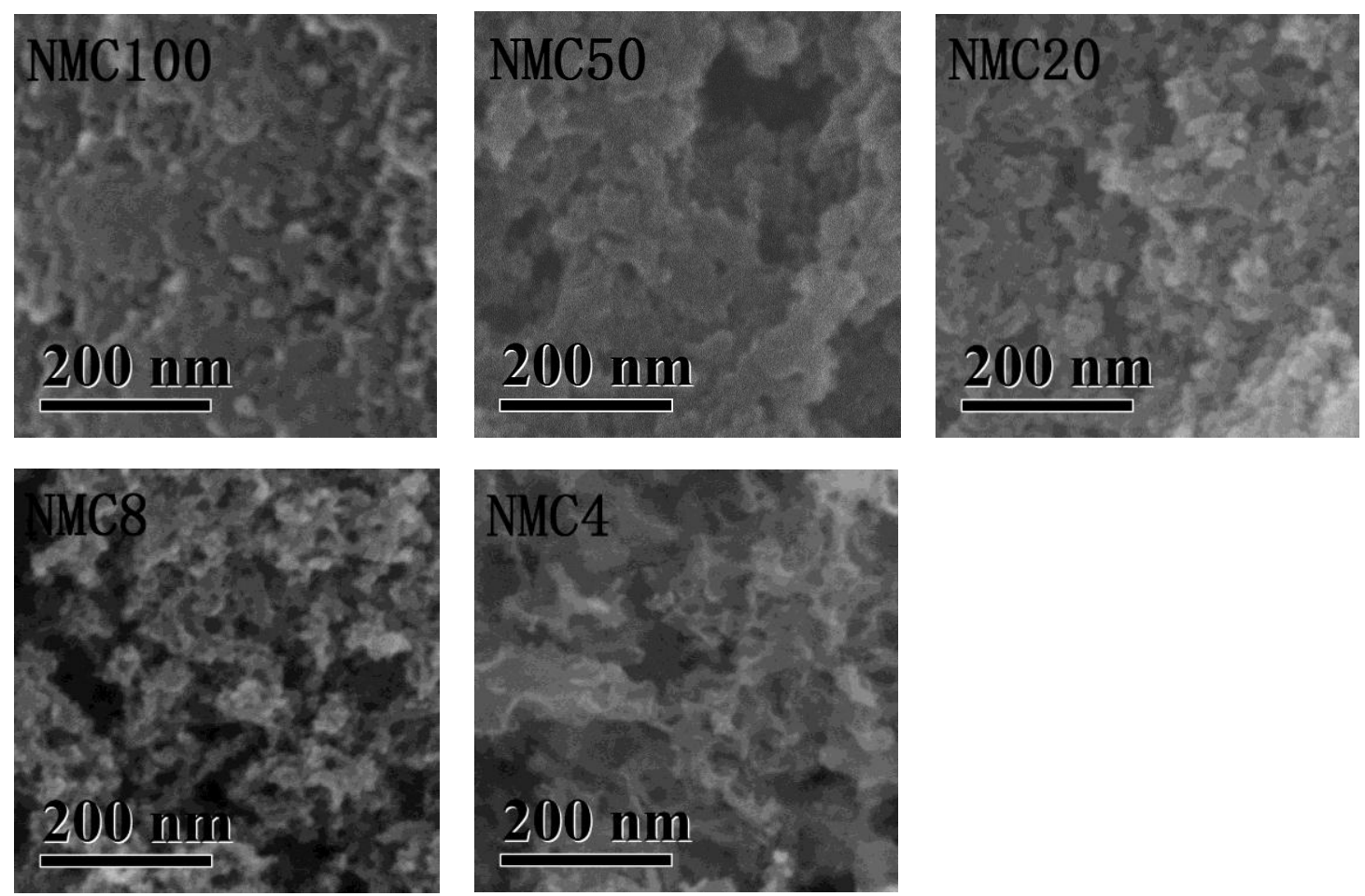

Figure 3. SEM images of the as-prepared NMCs 
Figure S1 (in the Supplementary Information) shows the FTIR spectra of NMC50 before and after $\mathrm{HF}$ etching. The disappeared characteristic peaks of $\mathrm{SiO}_{2}$ around 1100, 800 and $470 \mathrm{~cm}^{-1}$ after $\mathrm{HF}$ etching indicate the successful removal of $\mathrm{SiO}_{2}$ template, remaining abundant mesopores in the resulted NMC sample. TEM image of the as-prepared silica/nanocarbon composite with a TEOS/HF ratio of 50 (SiO2/NC50) shows that $\mathrm{SiO}_{2}$ gel network homogeneously disperses in the carbon framework, and presents a 3-D continuous skeleton structure (Figure S2), which allows us to construct continuous mesopore structure for the resulted NMCs. Figure 2 and 3 present the TEM and SEM images of the as-prepared NMCs, respectively. The samples present $3 \mathrm{D}$ continuous carbon skeleton and a number of continuous mesopores. These mesopores are interconnected very well to each other in all directions. Obviously, HF plays an important role in tailoring the microstructure morphology and mesopore diameter $\left(D_{P}\right)$ of $\mathrm{NMC}$, i.e., the pore size increases with increasing the HF content. This can be explained by the catalyzing effect of fluorine anion during both the hydrolysis and polymerization reactions (Figure S3 and S4). In brief, as increasing the fluorine anion amount, the size of the resulting sol particles will increase correspondingly to overcome the repulsive effect of surface charges built up on the fluorine anion-containing particulate clusters [30-31]. Therefore, silica skeleton presents an increasing size when increasing HF content, resulting in an increasing mesopore size of the as-prepared NMCs. 

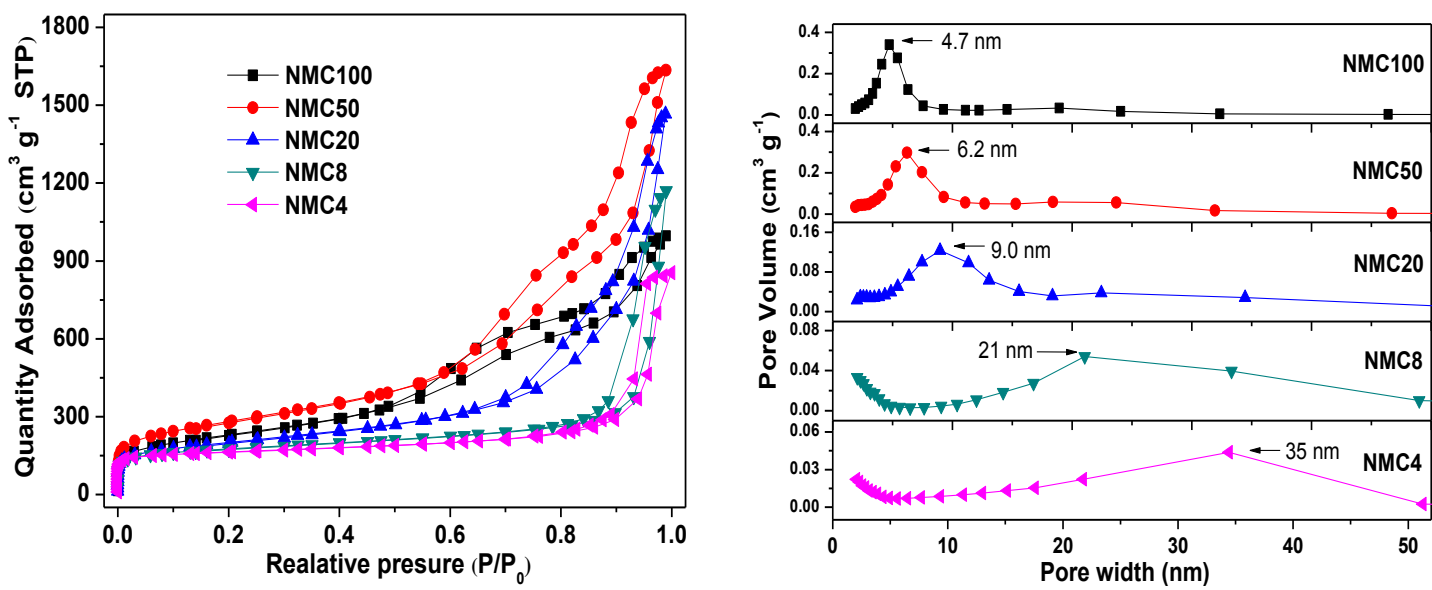

Figure 4. $\mathrm{N}_{2}$ adsorption-desorption isotherms (left) and BJH pore size distribution curves (right) of the as-prepared NMCs

Table 1 Pore structure parameters and nitrogen content of the as-prepared NMCs

\begin{tabular}{|c|c|c|c|c|c|c|}
\hline Sample & $\begin{array}{c}\text { SBET } \\
\left(\mathrm{m}^{2} \mathbf{g}^{-1}\right)\end{array}$ & $\begin{array}{c}V_{\text {mic }} \\
\left(\mathrm{cm}^{3} \mathrm{~g}^{-1}\right)\end{array}$ & $\begin{array}{c}V_{\text {mes }} \\
\left(\mathrm{cm}^{3} \mathrm{~g}^{-1}\right)\end{array}$ & $V_{\text {mes }} \%^{*}$ & $\begin{array}{c}\text { Nitrogen } \\
\text { content (wt.\%) }\end{array}$ & $\begin{array}{r}\mathbf{D}_{\mathbf{p}} \\
(\mathbf{n m})\end{array}$ \\
\hline NMC100 & 819 & 0.04 & 1.53 & $97.5 \%$ & 8.2 & 4.7 \\
\hline NMC50 & 1003 & 0.06 & 2.56 & $97.7 \%$ & 8.4 & 6.2 \\
\hline NMC20 & 704 & 0.08 & 2.28 & $96.6 \%$ & 8.4 & 9.0 \\
\hline NMC8 & 606 & 0.13 & 1.71 & $92.9 \%$ & 8.7 & 21 \\
\hline NMC4 & 556 & 0.16 & 1.18 & $88.1 \%$ & 8.6 & 35 \\
\hline
\end{tabular}

$* \mathrm{~V}_{\text {mes }} \%=\mathrm{V}_{\text {mes }} /\left(\mathrm{V}_{\text {mes }}+\mathrm{V}_{\text {mic }}\right) \times 100 \%$

Such a developed mesopore structure can be quantitatively confirmed from the obvious uptakes at medium or high relative pressure in the $\mathrm{N}_{2}$ adsorption-desorption isotherms (Figure 4 left). According to the pore size distribution curves by BJH theory, 
$\mathrm{D}_{\mathrm{P}}$ of the as-prepared NMC increases from 4.7 to $35 \mathrm{~nm}$ while increasing the HF/TEOS from 1/100 to $1 / 4$ (Figure 4 right), consisting with the TEM results. Due to the decreasing shrinkage ratio resulted from the increasing silica skeleton size, etching effect of excessive HF catalyst and decreasing trend of mesopore size, both the surface area and mesopore volume of NMCs increase first and then decrease (Table 1). As a result, NMC50 sample shows the highest $\mathrm{V}_{\text {mes }}$ of $2.56 \mathrm{~cm}^{3} \mathrm{~g}^{-1}$ and largest $\mathrm{S}_{\mathrm{BET}}$ of $1003 \mathrm{~m}^{2} \mathrm{~g}^{-1}$ with a mesopore size of $6.2 \mathrm{~nm}$. Its extremely developed $\mathrm{V}_{\text {mes }} \%$ of $97.7 \%$, to our knowledge, is the highest one reported so far for NCs in supercapacitor application. These small mesopores coupled with extremely developed mesoporosity are beneficial to constructing large ion-accessible surface area [32-34], namely, the increased surface utilization. Meanwhile, all the samples show similar nitrogen content of about $8.5 \mathrm{wt} . \%$ owing to the same carbonization temperature. One can prospect that such a large surface area arisen from the high mesoporosity will make NMC particularly enticing for energy storage in supercapacitors, because it can facilitate better diffusion and transport of electrolyte, increasing both ion accessible surface area for charge accumulation and NFGs utilization. As a proof-of-concept demonstration, we carried out electrochemical measurements for the as-prepared samples.

Figure 5a shows the galvanostatic charge-discharge curves of NMCs at $0.1 \mathrm{~A} \mathrm{~g}^{-1}$. Obviously, because of the comparable nitrogen content, discharge time of the samples increases with the increasing surface area. With the longest discharge time, NMC50 demonstrates the highest $\mathrm{C}_{\mathrm{g}}$ of $325 \mathrm{~F} \mathrm{~g}^{-1}$. The calculated $\mathrm{C}_{\mathrm{g}}$ of NMC100, NMC20, 
NMC8 and NMC4 are 285, 252, 223 and $210 \mathrm{~F} \mathrm{~g}^{-1}$, respectively. Meanwhile, all of the samples present low charge transfer resistance (lower than $1 \Omega$ ), which can be estimated from the semicircle in high-frequency region of Nyquist plots (Figure 5b). As we mentioned above, because of the large ion accessible surface area arisen from the 3D continuous mesopore structure combining with the extremely high mesoporosity at high charge-discharge rates, these samples show excellent high-rate performance (Figure 6). For instance, the capacitance retention of all the samples stays above $88 \%$ when the current density increases by 100 times (at $10 \mathrm{~A} \mathrm{~g}^{-1}$ ). This value is much higher than those of other reported NCs (Table 2), including those highlighted as high-rate performance carbons [28, 35-38].
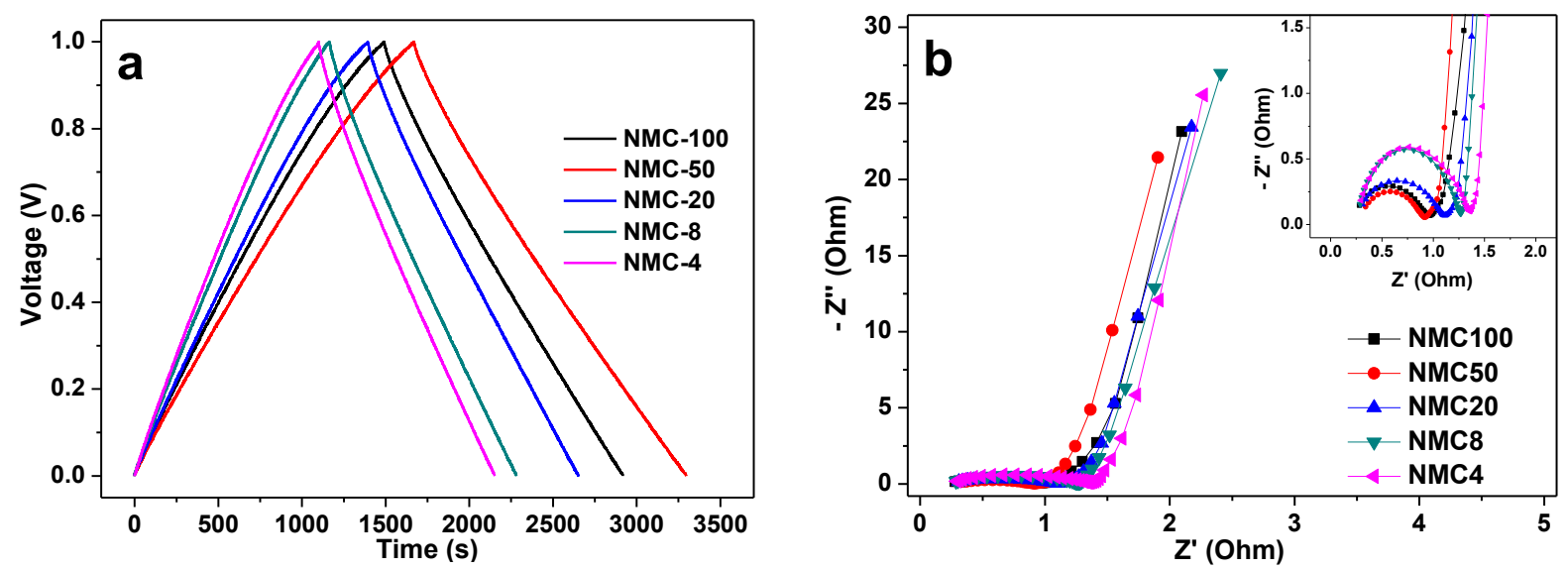

Figure 5. a) Galvanostatic charge-discharge curves at $0.1 \mathrm{Ag}^{-1}$ and b) Nyquist plots of the as-prepared NMCs. The inset shows the expanded high-frequency region of Nyquist plots. 
Table 2 High-rate performances of other reported NCs [11-13, 18, 26-29, 35-40].

\begin{tabular}{|c|c|c|c|c|c|c|}
\hline & Lower rate & & Higher rate & & Capacity & \\
\hline Nitrogen-enriched Sample & $\left(\mathrm{A} \mathrm{g}^{-1}\right)$ & $\mathrm{C}_{\mathrm{g}}\left(\mathrm{Fg}^{-1}\right)$ & $\left(\mathrm{A} \mathrm{g}^{-1}\right)$ & $\mathrm{C}_{\mathrm{g}}\left(\mathrm{Fg}^{-1}\right)$ & retention & Reference \\
\hline NMC50 & 0.1 & 325 & 10 & 286 & $88 \%$ & This study \\
\hline Microporous activated carbon & 0.05 & $\sim 230$ & 1 & $\sim 200$ & $87 \%$ & 11 \\
\hline Carbon aerogel & 0.1 & 208 & 2.5 & $\sim 177$ & $85 \%$ & 12 \\
\hline Hierarchical carbon & 0.2 & 325 & 10 & $\sim 190$ & $58 \%$ & 13 \\
\hline Graphene aerogels & 0.2 & 223 & 10 & 176 & $79 \%$ & 18 \\
\hline Porous carbon cryogel & 0.1 & 242 & 10 & 161 & $67 \%$ & 26 \\
\hline Carbon microspheres & 1 & 228 & 5 & $\sim 200$ & $88 \%$ & 27 \\
\hline Hierarchical porous carbon & 0.4 & 258 & 10 & 205 & $79 \%$ & 28 \\
\hline Porous carbon nanofiber & 0.2 & 302 & 10 & $\sim 220$ & $73 \%$ & 29 \\
\hline Hierarchical porous carbon & 0.2 & 260 & 10 & $\sim 170$ & $65 \%$ & 35 \\
\hline Carbon nanosheets & 0.5 & 187 & 10 & 129 & $69 \%$ & 36 \\
\hline Hollow carbon spheres & 0.5 & 213 & 10 & 118 & $55 \%$ & 37 \\
\hline Porous carbon & 0.2 & 280 & 10 & 171 & $60 \%$ & 38 \\
\hline Microporous carbon & 0.1 & 286 & 2 & 208 & $73 \%$ & 39 \\
\hline Porous carbon aerogel & 0.2 & 197 & 10 & 108 & $.55 \%$ & 40 \\
\hline
\end{tabular}




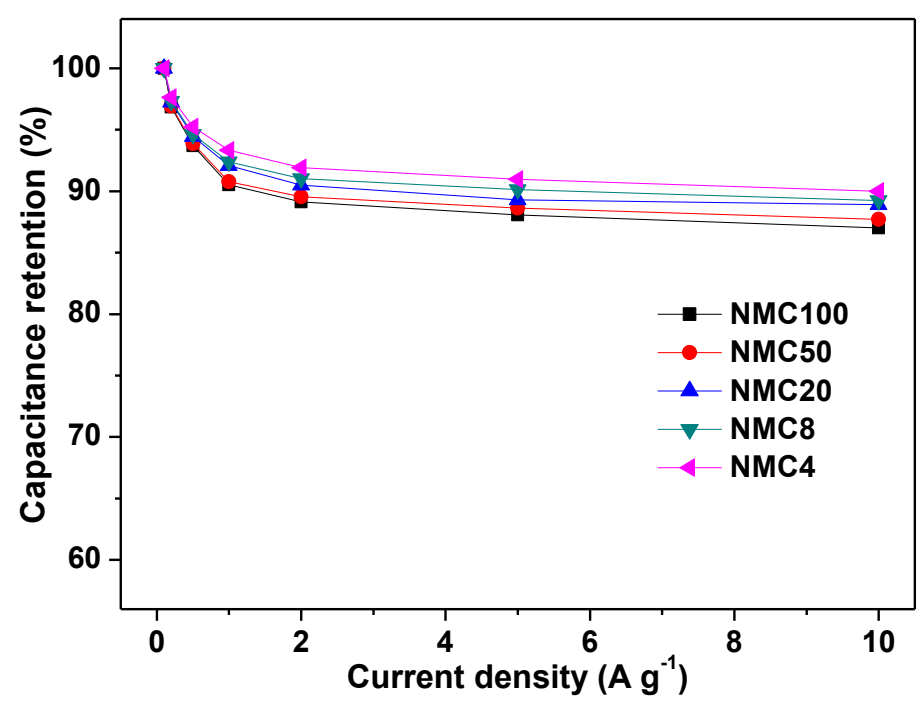

Figure 6. Capacitance retention at different current densities of NMCs.

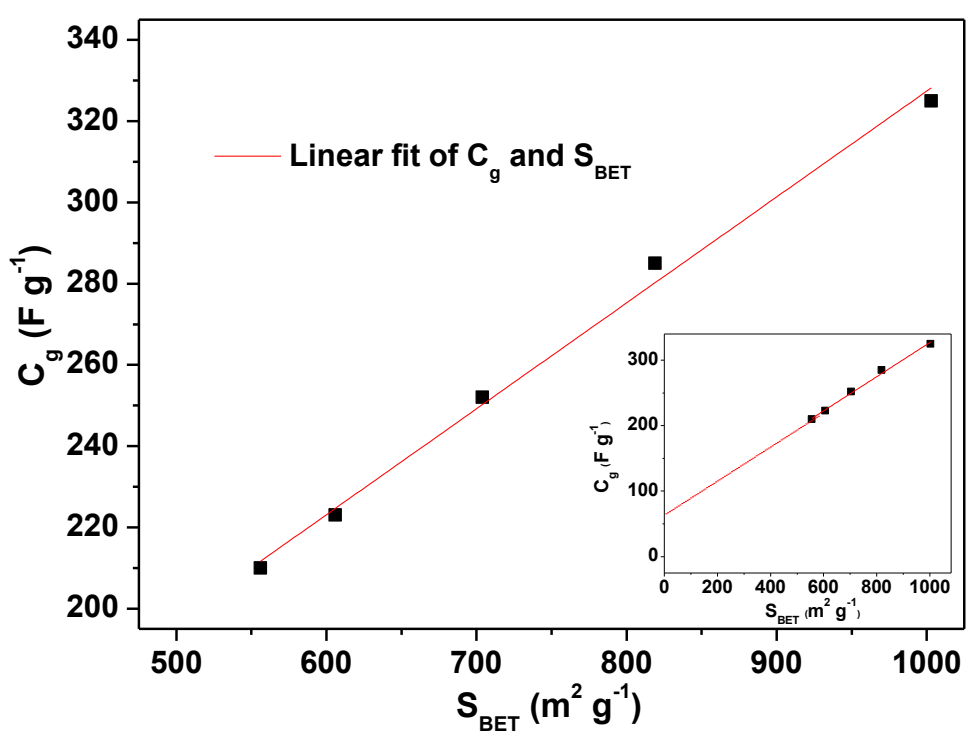

Figure 7. Linear relationship between $C_{g}$ and $S_{\text {BET. }}$. The inset shows the intercept of the extension line of fitting line.

Although a large surface area is considered necessary for a high energy density electrode material, $\mathrm{C}_{\mathrm{g}}$ is not directly proportional to surface area in most investigations owing to the low mass transfer efficiency of porous carbons with high surface area, especially that arisen from micropores. Thus, inevitably low $\mathrm{C}_{\mathrm{S}}$ of 10-25 $\mu \mathrm{F} \mathrm{cm} \mathrm{cm}^{-2}$ is usually obtained $[12-14,16,21,24-26]$. To our delight, in this research, $\mathrm{C}_{\mathrm{g}}$ 
of each sample shows good linear relationship with $\mathrm{S}_{\mathrm{BET}}$ (Figure 7), and their calculated $\mathrm{C}_{\mathrm{S}}$ is up to $32.4 \mu \mathrm{F} \mathrm{cm}{ }^{-2}$, confirming the excellent mass transfer capability of the as-prepared NMC unambiguously from another point of view. Based upon this, a pseudocapacitance of about $65 \mathrm{~F} \mathrm{~g}^{-1}$ can be estimated from the intersecting point between the extension line of fitting line and the $\mathrm{X}$-axis (Figure 7 inset), which is comparable with that calculated from discharge curves we presented previously [19].
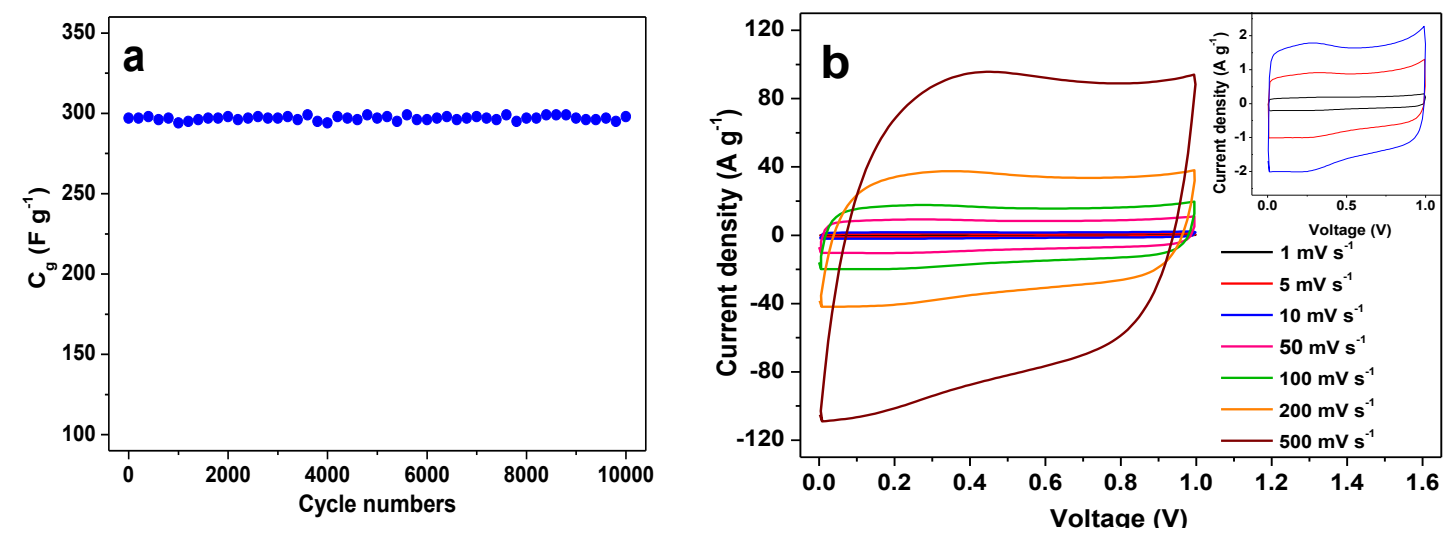

Figure 8. a) Cycle stability of NMC50 and b) CV curves of NMC50 at different scan rates. The inset shows the $\mathrm{CV}$ curves at relatively low scan rate of 1,5 and $10 \mathrm{mV} \mathrm{s}^{-1}$.

The excellent electrochemical performance of NMC50 can be confirmed by cycling stability test and CV measurements in Figure 8. NMC50 exhibits extraordinarily high cycle stability with little degradation during 10000 cycles at $1 \mathrm{~A} \mathrm{~g}^{-1}$ (Figure 8a), indicative of a robust architecture of the 3D continuous carbon framework during the repeated charging/discharging process. On the other hand, the ability of ion diffusion/transfer within the nanocarbon structure can be estimated from the rectangular shape of the CV curves generally [41]. It can be observed from the CV curves that NMC50 shows near-rectangular shapes (Figure 8b), even at high scan 
rates. These results clearly highlight the superior supercapacitive behavior, benefitting from its developed mesoporous nanostructures. The developed carbon skeleton, continuous mesoporous structures and high mesoporosity will guarantee rapid transfer/diffusion of electron and electrolyte ions, respectively, whereas the high surface area will provide many active sites for charge accumulation. Moreover, NFGs can also enhance electrolyte wettability and provide additional pseudocapacitance.

\section{Conclusions}

A simple methodology based on the silica-skeleton-tailoring effect of HF has been successfully developed to prepare NMCs with tunable mesopore size. By varying the HF/TEOS ratio, mesopore size of the as-prepared NMCs can be easily controlled in a wide range of $4.7-35 \mathrm{~nm}$. Benefiting from the 3D continues mesoporous structure, extremely high mesoporosity of $97.7 \%$ and large surface area of $1003 \mathrm{~m}^{2} \mathrm{~g}^{-1}$, NMC50 sample presents the highest $\mathrm{C}_{\mathrm{g}}$ of $325 \mathrm{~F} \mathrm{~g}^{-1}$ at $0.1 \mathrm{~A} \mathrm{~g}^{-1}$, which can stay over $88 \%$ (286 $\left.\mathrm{F} \mathrm{g}^{-1}\right)$ as the current density increases by 100 times $\left(10 \mathrm{~A} \mathrm{~g}^{-1}\right)$. We hope that this new strategy of constructing tunable mesopore for NMCs may open new opportunities to further optimize the electrochemical performance as well as expand practical application fields such as selective adsorption and catalyst support.

\section{Acknowledgements}

This research was financially supported by The Start-up funding for Young talent of Guangdong University of technology (220413521), National Science Foundation for 
Post-doctoral Scientists of China (2013M542153), Scientific and technological innovation project for small and medium technology enterprises of Shunde District (2014CX001), Scientific and technological project of Administration of Quality and Technology Supervision of Guangdong Province (2015PJ03).

\section{References}

[1] D. Bhattacharjya, H. Park, M. Kim, H. Choi, S.N. Inamdar, J. Yu, Langmuir 30 (2014) 318-324.

[2] L. Qie, W. Chen, Z. Wang, Q. Shao, X. Li, L. Yuan, X. Hu, W. Zhang, Y. Huang, Adv. Mater. 24 (2012) 2047-2050.

[3] J. Ou, Y. Zhang, L. Chen, Q. Zhao, Y. Meng, Y. Guo, D. Xiao, J. Mater. Chem. A 3 (2015) 6534-6341.

[4] S.M. Unni, S.N. Bhange, R. Illathvalappil, N. Mutneja, K.R. Patil, S. Kurungot, Small 11 (2015) 352-362.

[5] Y. Tang, B.L. Allen, D.R. Kauffman, A. Star, J. Am. Chem. Soc. 131 (2009) $13200-13201$.

[6] L. Chen, Z. Huang, H. Liang, Q. Guan, S. Yu, Adv Mater 25 (2013) 4746-4752.

[7] L. Chen, Z. Huang, H. Liang, W. Yao, Z. Yu, S. Yu, Energ. Environ. Sci. 6 (2013) $3331-3338$.

[8] D.W. Wang, F. Li, L.C. Yin, X. Lu, Z.G. Chen, I.R. Gentle, G.Q. Lu, H.M. Cheng, Chem-Eur. J. 18 (2012) 5345-5351.

[9] Y. Li, J. Dong, J. Zhang, X. Zhao, P. Yu, L. Jin, Q. Zhang, Small 11 (2015) 
3476-3484.

[10] Z.J. Li, W. Lv, C. Zhang, B.H. Li, F.Y. Kang, Q.H. Yang, Carbon 92 (2015) $11-14$

[11] D. Hulicova-Jurcakova, M. Seredych, G.Q. Lu, T.J. Bandosz, Adv. Funct. Mater. 19 (2009) 438-447.

[12] M. Wahid, G. Parte, D. Phase, S. Ogale, J. Mater. Chem. A 3 (2015) 1208-1215.

[13] Y. Song, S. Hu, X. Dong, Y. Wang, C. Wang, Y.A. Xia, Electrochim. Acta 146 (2014) 485-494.

[14] A. Chen, Y. Yu, T. Xing, R. Wang, Y. Li, Y. Li, Mater. Lett. 157 (2015) 30-33.

[15] D. Zhang, L. Zheng, Y. Ma, L. Lei, Q. Li, Y. Li, H. Luo, H. Feng, Y. Hao, ACS Appl. Mater. Inter. 6 (2014) 2657-2665.

[16] K. Kang, S. Hong, B. Lee, J. Lee, Electrochem. Commun. 10 (2008) 1105-1108.

[17] J. Ludwinowicz, M. Jaroniec, Carbon 94 (2015) 673-679.

[18] Z. Sui, Y. Meng, P. Xiao, Z. Zhao, Z. Wei, B. Han, ACS Appl. Mater. Inter. 7 (2015) 1431-1438.

[19] X.Q. Yang, D.C. Wu, X.M. Chen, R.W. Fu, J. Phys. Chem. C 114 (2010) $8581-8586$.

[20] W. Kim, M. Kang, J. Joo, N. Kim, I. Song, P. Kim, J. Yoon, J. Yi, J. Power Sources 195 (2010) 2125-2129.

[21] Q. Shi, R.Y. Zhang, Y.Y Lv, Y.H. Deng, A.A. Elzatahrya, D.Y Zhao, Carbon 84 (2015) 335-346.

[22] G.P. Mane, S.N. Talapaneni, C. Anand, S. Varghese, H. Iwai, Q. Ji, K. Ariga, T. 
Mori, A. Vinu, Adv. Funct. Mater. 22 (2012) 3596-3604.

[23] D. Hulicova, M. Kodama, H. Hatori, Chem. Mater. 18 (2006) 2318-2326.

[24] H. Liu, H. Song, X. Chen, S. Zhang, J. Zhou, Z. Ma, J. Power Sources 285 (2015) 303-309.

[25] J.H. Jiang, Q.M. Gao, K.S. Xia, J. Hu, Micropor. Mesopor. Mat. 118 (2009) 28-34.

[26] Z. Ling, C. Yu, X. Fan, S. Liu, J. Yang, M. Zhang, G. Wang, N. Xiao, J Qiu, Nanotechnology 23 (2015) 374003. doi:10.1088/0957-4484/26/37/374003.

[27] D. Zhu, Y. Wang, L. Gan, M. Liu, K. Cheng, Y. Zhao, X. Deng, D. Sun, Electrochim. Acta 158 (2015) 166-174.

[28] G. Wang, J. Zhang, S. Kuang, J. Zhou, W. Xing, S. Zhuo, Electrochim. Acta 153 (2015) 273-279.

[29] K. Huang K, M. Li, Z. Chen, Y. Yao, X. Yang, Electrochim. Acta 158 (2015) 306-313.

[30] E.J.A Pope, J.D.J Mackenzie, J. Non-Cryst. Solids 87 (1986) 185-198.

[31] S.S. Wang, H. L. Liu, L.Y Zhang, X. Yao, Chinese J. Mater. Res. 10 (1996) 183-186.

[32] C.F. Li, X.Q. Yang, G.Q. Zhang, Mater. Lett. 161 (2015) 538-541.

[33] W.J. Gao, Y. Wan, Y.Q. Dou, D.Y. Zhao, Adv. Energy Mater. 1 (2011) 115-123.

[34] Y.P. Zhai, Y.Q. Dou, X.X. Liu, S.S. Park, C.S. Ha, D.Y. Zhao, Carbon 49 (2011) $545-555$.

[35] J. Zhou, Z. Zhang, W. Xing, J. Yu, G. Han, W. Si, S. Zhuo, Electrochim. Acta 153 
(2015) 68-75.

[36] H. Peng, G. Ma, K. Sun, J. Mu, Z. Lei, J. Mater. Chem. A 2 (2014) 17297-17301.

[37] J. Han, G. Xu, B. Ding, J. Pan, H. Dou, D.R. MacFarlane, J. Mater. Chem. A 2 (2014) 5352-5357.

[38] J. Jiang, L. Bao, Y. Qiang, Y. Xiong, J. Chen, S. Gu, J. Chen, Electrochim. Acta 158 (2015) 229-236.

[39] S. Zhong, C. Zhan, D. Cao, Carbon 85 (2015) 51-59.

[40] P. Hao, Z. Zhao, Y. Leng, J. Tian, Y. Sang, R.I. Boughton, C.P. Wong, H. Liu, B. Yang, Nano Energy 15 (2015) 9-23.

[41] H. Zhong, F. Xu, Z.H. Li, R.W. Fu, D.C. Wu, Nanoscale 5 (2013) 1-5. 